\section{Revista Chilena de Pediatría}

www.revistachilenadepediatria.cl

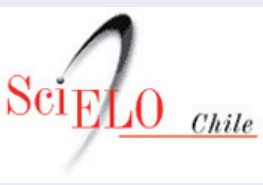

www.scielo.cl

\title{
Diagnóstico en medicina en la era de las "omicas"
}

\section{Diagnostics in medicine in the "omics" era}

\author{
Mauricio J. Farfán ${ }^{\mathrm{a}, \mathrm{b}}$ y Juan Pablo Torres ${ }^{\mathrm{a}}$ \\ aDepartamento de Pediatría y Cirugía Infantil, Campus Oriente, \\ Facultad de Medicina, Universidad de Chile

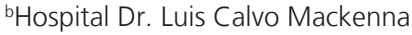

El diagnóstico de laboratorio está en constante desarrollo. El conocimiento generado por las ciencias básicas, los desafios clínicos y el desarrollo de nuevas tecnologías, han confluido en un incremento significativo de técnicas de diagnóstico más rápidas, sensibles y específicas, lo que ha llevado a plantear una aproximación más personalizada de las patologías, denominada como medicina de precisión. De forma general, la medicina de precisión integra los datos clínicos, anatomopatológicos y moleculares con el fin de seleccionar el tratamiento adaptado al perfil biológico de un individuo. El desarrollo de las tecnologías "omicas", definidas por la naturaleza de las moléculas que son producidas por una células, tejido u organismo han demostrado avances promisorios para el diagnóstico, manejo y pronóstico de diversas enfermedades, proyectándose como un pilar fundamental en la implementación de la medicina de precisión. La detección de genes (genómica), ARN (transcriptómica), proteínas (proteómica) y los metabolitos (metabolómica) en una muestra, ha llevado al diagnóstico molecular a un nivel de especialización extraordinario que lentamente se ha ido integrado a la práctica clínica. Sin embargo, es importante conocer sus alcances, limitaciones y los desafíos en su implementación para el manejo de los pacientes.

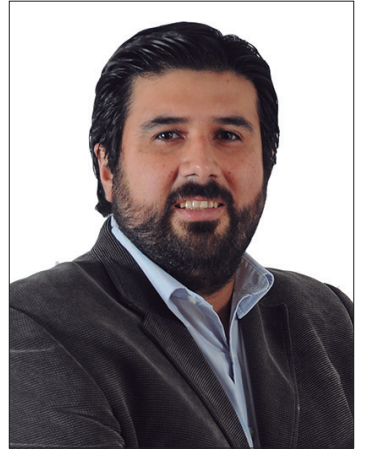

Mauricio J. Farfán

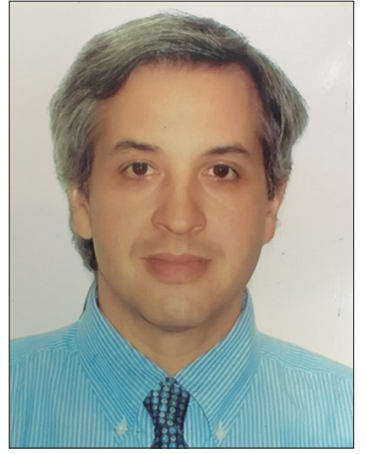

Juan Pablo Torres

\section{Genómica}

La genómica, definida por el estudio del genoma de un individuo ha sido la tecnología "omica" de mayor desarrollo. El acceso a técnicas de secuenciación masiva de alto rendimiento y bajo costo ha tenido un gran impacto en el diagnóstico de patologías, especialmente el cáncer. Hoy en día numerosos centros y compañías ofrecen servicios de secuenciación de ADN como herramienta diagnóstica o de seguimiento de neoplasias como cáncer de mama, próstata, leucemias, por nombrar algunos. Estos servicios van acompañados de información para el clínico sobre los hallazgos encontrados de forma de guiar el manejo de la neoplasia. Si bien el desarrollo de técnicas de diagnóstico molecular como la reacción de polimerasa en cadena en tiempo real (RPC-TR) permiten la detección de mutaciones genéticas asociadas a algunas neoplasia, la genómica ofrece una cobertura más amplia, incluyendo todas las mutaciones posibles incluidas las más comunes, lo que abre un horizonte para neoplasias que sin diagnosticadas $^{1}$.

Otra de las áreas donde la genómica ha tenido un impacto significativo es el estudio de la microbiota. El uso de técnicas de secuenciación masiva ha mostrado ser una herramienta tremendamente útil para deter- 
minar los microorganismos que componen un sitio anatómico particular. Hoy en día numerosas patologías se han asociado a cambios en la microbiota, lo que ha llevado a plantear su uso como en la medicina de precisión como una herramienta de diagnóstico para determinar estos cambios ${ }^{2}$. Recientemente, en nuestro país ya se han ido generando líneas de investigación relacionadas al estudio de la microbiota intestinal en niños con diarrea (FONDECYT Regular 1160426) y la $^{3}$ microbiota respiratoria en niños inmunocomprometidos con infecciones respiratorias virales (FONDECYT Regular 1171795), entre otras.

El diagnóstico de infecciones de manera ultra rápida a través de secuenciación masiva también ha sido un área de desarrollo emergente, permitiendo la detección de patógenos habituales e inhabituales en muestras clínicas, en un tiempo incluso menor a las $24 \mathrm{~h}^{4}$.

\section{Transcriptómica}

Para que una proteínas se produzca, es necesario que el gen que la codifica se exprese, mediante un ARN mensajero (ARNm) que actúa como una molécula puente entre el gen y la proteína. En este sentido, la cuantificación de la expresión génica es una manera indirecta de determinar la expresión de un gen en particular. La transcriptómica se define como la detección y/o cuantificación de los ARN en una célula u organismo, que refleja los genes que activamente se están expresados en un momento determinado. El uso de técnicas de PCR-TR, microarreglos y secuenciación de ARN (RNA-seq) son las técnicas que más utilizadas, siendo alguna de ellas utilizadas hoy en día en la practica clínica, principalmente para el diagnóstico y monitorización de cáncer ${ }^{5}$, así como para el diagnóstico de infecciones virales y bacterianas en niños ${ }^{6}$. En Chile, se han comunicado estudios transcripcionales en niños con infección persistente por Helicobater pylori, permitiendo el estudio de potenciales genes asociados a mayor severidad de la infección y su posible asociación con el desarrollo de cáncer en años posteriores ${ }^{7}$.

Otra de las aplicaciones de la transcriptómica se encuentra la detección de virus ARN. Debido a la alta tasa de mutaciones de estos microorganismos (virus influenza) o su elevada mortalidad (virus ébola), la transcriptómica ofrece una alternativa donde los test diagnósticos moleculares clásicos no son lo suficientemente específicos o aún no han sido desarrollados ${ }^{8}$.

\section{Proteómica}

El proteoma se ha definido como el set de proteínas presentes en una célula, tejido y organismo. La proteómica tiene como objetivo caracterizar las proteínas presentes como origen o consecuencia de una patología. Si bien existen numerosos marcadores proteicos asociados al desarrollo y progresión de enfermedades, la proteómica ofrece la posibilidad de analizar una mayor cantidad de proteínas e identificar nuevos biomarcadores. Las técnica de espectrometría de masas, en sus diferentes versiones, han sido relevantes en la identificación de nuevos biomarcadores para el diagnóstico y predicción de riesgo en cáncer y enfermedades cardiacas y neurodegenerativas ${ }^{9,10,11}$.

Actualmente, una de las técnicas de mayor impacto en clínica es la aplicación de MALDI-TOF en el diagnóstico microbiológico. La capacidad de identificar un microorganismo en una colonia en un par de minutos ha optimizado el diagnóstico microbiológico clásico, llevándolo a una nueva era ${ }^{12}$.

\section{Metabolómica}

Los metabolitos se definen como los productos de reacciones bioquímicas que tiene a lugar en el organismo. A diferencias de las otras tecnología "omicas", la naturaleza de las moléculas que se analizan en los estudios de metabolómica son más complejas, encontrándose lípidos, carbohidratos, proteínas, glicoproteínas, entre otras. Debido a que el metaboloma es la consecuencia de los cambios expresión de genes y actividad de las proteínas que codifican, los cambios en el metaboloma dan información relevante sobre los procesos bioquímicos que ocurren en una patología determinada. Nuevamente, es en el cáncer donde el uso de la metabolómica ha entregado resultados sobre metabolitos con el potencial de ser utilizados como biomarcadores para el diagnóstico, monitorización y respuesta al tratamiento. Por ejemplo, en la búsqueda de metabolitos que permitan discriminar pacientes con cáncer de mama, los análisis de metabolómica en saliva permitieron detectar que la razón de poliaminas presentes permite discriminar entre pacientes con cáncer e individuos sanos ${ }^{13}$. Ejemplos como éste, permiten proyectar el uso de la metabolómica como una herramienta con alto potencial en la práctica clínica.

Las tecnologías "omicas" han mostrado ser tremendamente útiles en la generación de nuevo conocimiento en medicina, permitiendo el desarrollo aún incipiente de una medicina cada vez más personalizada, sin embargo, su aplicación en la practica clínica aún es limitada. La mayoría de estas técnicas requieren de una infraestructura y equipamiento de alto nivel. Sin embargo, el aumento de aplicaciones y el impacto en el diagnostico y manejo de patologías, ha hecho que estas tecnologías sea más accesibles. Uno de los puntos claves en su implementación como herramienta diagnós- 
tica es el desarrollo de unidades de bioinformática en los centros de salud, apoyando directamente al clínico en la interpretación de los resultados. Considerando la importancia de las técnicas "omicas" en medicina, es importante conocer este tipo de técnicas y sus aplicaciones, ya que su implementación en la practica clínica, como parte de la medicina de precisión, ya es una realidad y posiblemente tendrá un desarrollo exponencial en los próximos años.

\section{Financiación}

Financiado por FONDECYT Regular 1160426 (MJF) 1171795 (JPT)

\section{Conflicto de intereses}

Los autores declaran no tener ningún conflicto de intereses.

\section{Referencias}

1. Nakagawa H, Fujita M. Whole genome sequencing analysis for cancer genomics and precision medicine. Cancer Sci. 2018;109(3):513-22.

2. Kashyap PC, Chia N, Nelson H, Segal E, Elinav E. Microbiome at the Frontier of Personalized Medicine. Mayo Clin Proc. 2017;92(12):1855-64.

3. Gallardo P, Izquierdo M, Vidal RM, et al. Distinctive Gut Microbiota Is Associated with Diarrheagenic Escherichia coli Infections in Chilean Children. Front Cell Infect Microbiol. 2017;7:424.

4. Naccache SN, Federman S, Veeraraghavan $\mathrm{N}$, et al. A cloud-compatible bioinformatics pipeline for ultrarapid pathogen identification from nextgeneration sequencing of clinical samples. Genome Res. 2014;24(7):1180-92.

5. Byron SA, Van Keuren-Jensen KR, Engelthaler DM, Carpten JD, Craig DW.
Translating RNA sequencing into clinical diagnostics: opportunities and challenges. Nat Rev Genet. 2016;17(5):257-71.

6. Cruz AT, Mahajan P, Bonsu BK, et al. Accuracy of Complete Blood Cell Counts to Identify Febrile Infants 60 Days or Younger With Invasive Bacterial Infections. JAMA Pediatr. 2017;171(11):e172927.

7. O’Ryan ML, Lucero Y, Rabello M, et al. Persistent and transient Helicobacter pylori infections in early childhood. Clin Infect Dis. 2015;61(2):211-8.

8. Gire SK, Goba A, Andersen KG, et al. Genomic surveillance elucidates Ebola virus origin and transmission during the 2014 outbreak. Science. 2014;345(6202):1369-72.

9. Moris D, Ntanasis-Stathopoulos I, Tsilimigras DI, Adam MA, Yang C-FJ, Harpole D, Theocharis S.Insights into Novel Prognostic and Possible Predictive Biomarkers of Lung Neuroendocrine
Tumors. Cancer Genomics Proteomics. 2018;15(2):153-163

10. Israr MZ, Heaney LM, Suzuki T. Proteomic Biomarkers of Heart Failure. Heart Fail Clin. 2018;14(1):93-107.

11. Cagnone M, Bardoni A, Iadarola P, Viglio S. Could Proteomics Become a Future Useful Tool to Shed Light on the Mechanisms of Rare Neurodegenerative Disorders?. High Throughput. 2018;7(1). pii: E2.

12. van Belkum A, Welker M, Pincus D, Charrier JP, Girard V. Matrix-Assisted Laser Desorption Ionization Time-ofFlight Mass Spectrometry in Clinical Microbiology: What Are the Current Issues?. Ann Lab Med. 2017;37(6):475-83.

13. Takayama T, Tsutsui H, Shimizu I, et al. Diagnostic approach to breast cancer patients based on target metabolomics in saliva by liquid chromatography with tandem mass spectrometry. Clin Chim Acta. 2016;452:18-26. 